\title{
A DFT Study of the Strain Effect on the Optical Properties of Linear and Dimerized Titanium Nanochains
}

\author{
Z. SOHRABIKIA AND M. JAFARI* \\ Department of Physics, K.N. Toosi University of Technology, P.O. Box 16315-1618, Tehran, Iran \\ (Received July 15, 2015)
}

Optical properties of both linear and dimerized nanochains of titanium at different atomic distances are calculated using the full potential linearized augmented plane wave plus local orbital method, and using the generalized gradient approximation. When Ti nanochains were compressed, the position of critical points such as static dielectric constants and the main peaks in optical spectra shifted with an increased or decreased energy comparative to that at equilibrium constants. Under tensile strain $\varepsilon_{1 \max }(\omega)$ decreases in linear and dimerized structures. The plasma frequency for both structures decreases as the bond length increases. Moreover, the peaks of the energy loss function move toward higher energies with increasing bond length for linear structure, while they do not change significantly for the dimerized structure. The absorption for both nanostructures decreases by increasing the bond lengths.

DOI: 10.12693/APhysPolA.129.344

PACS/topics: 71.15.Mb, 78.67.Uh, 62.23.Hj

\section{Introduction}

Fabricating monoatomic nanowires (NWs) is possible nowadays using advanced technology [1]. There are different experimental techniques that make it possible to growth nanostructured systems with desired dimensionality. These atomic chains which are used in nanodevices were created using systems such as carbon nanotubes [2], directed assembly [3], or self-assembly on stepped surfaces $[4,5]$. It has been proved that changing the dimension of a system usually causes new physics: one-dimensional metal have a very different behavior to two- or three-dimensional metals. Recently a systematic $a b$ initio studies of the magnetic and electronic properties of $3 d$ transition metal (TM) nanowires suggested that all $3 d \mathrm{TM}$ in both linear and zigzag structures have a stable or metastable ferromagnetic (FM) state [6,7].

Titanium as one of the TM elements with a partially filled $3 d$ shell has shown specific and exceptional behaviors in either bulk or nanowire forms. Large experimental investigations on $\mathrm{Ti}$ nanostructures have been done [819]. Nakamura et al. fabricated titanium nanowires in sapphire by utilizing lattice dislocations which exhibited excellent electrical conductivity [8]. Short suspended nanowires were fabricated by sweeping the metallic surface by the tip of a scanning tunneling microscope [9]. Scanning tunneling microscopy and scanning tunneling spectroscopy (STM/STS) of a titanium (Ti) amorphous film and titanium oxide dots were investigated at room temperature and ambient air by scanning tunneling microscope (STM) [10]. Fabrication of these nanostructures was also done by methods like electro deposition on paralytic graphite [16]. Pendelton and his group synthesized

*corresponding author; e-mail: jafari@kntu.ac.ir the nanochains of titanium using crown ether as a template [15]. Other experimental studies on fabricating titanium nanostructures have been done on aluminum oxide [16] and by $\mathrm{Ga}+$ focused ion beam [17]. Continuous wires of titanium atoms deposited on single wall carbon nanotube were formed by Zhang et al. [20, 21].

Beside experimental efforts, theoretical calculations of electronic, magnetic, and optical properties of titanium nanochains have been carried out using methods such as molecular dynamics, semi-empirical tight binding, or $a b$ initio density functional theory. At first, some simulations were manifested by the Monte Carlo approach [22]. Li et al. studied different structures of titanium nanochains in nonmagnetic state and found the zigzag structure as the most stable one [23]. Computational effort in this direction was led by the pioneering work of Zhu et al., who predicted the emergence of magnetism in titanium nanochains [24]. They found the equilibrium bond length and magnetic moment for different bond lengths of monatomic wire of Ti. Magnetic properties of $3 d \mathrm{TM}$ chains on vicinal $\mathrm{Cu}$ (111) surface were presented by Hashemi et al. [25]. Moreover, electronic and magnetic properties of $\mathrm{Ti}$ monolayers were investigated in both nonmagnetic (NM) and ferromagnetic (FM) states [26]. Ataca et al. put an emphasis on studying infinite periodic linear, dimerized, and zigzag titanium nanochains as well as finite short segments [7]. Bala et al. performed an informative work on titanium nanochains in NM, FM, and also antiferromagnetic (AFM) states [27]. They suggested that, among all Ti nanochains, at larger bond length values, dimerized structure was more stable and FM state was more favorable than AFM state.

The purpose of the present work is to make a systematic ab initio study of the optical properties of linear and dimerized titanium nanochains at different bond lengths. The $a b$ initio calculations can give tan idea 
about properties well before choosing the material for any application. Optical properties of solids [28] are of wide interest because of their diverse applications in industrial and optoelectronic devices [29, 30]. The imaginary and real parts of dielectric function, energy loss function, and optical conductivity are also calculated to understand the effect of nanochains bond length on these properties.

\section{Computational setup}

Our results for linear and dimerized titanium nanochains are obtained using the all electron full potential linearized augmented plane wave (FPLAPW) method [31]. The exchange and correlation effects are treated using the generalized gradient approximation (GGA) [32, 33] with the formal Perdew-Burke-Ernzerhof (PBE) parameterization scheme [34-36]. Core states are treated fully relativistically with the inclusion of local orbitals for semi-core states $(3 s, 3 p)$. For the valence states $(3 d, 4 s)$, scalar relativistic approach is used, because for $3 d$ transition metals, spin-orbit coupling does not have any significant effect on electronic and magnetic properties of the system [6]. For calculating the total energy of atoms, supercell approached is used and the distance between the atomic wires is taken to be about $10 \AA$, which is sufficient to eliminate the inter-wire interactions. In order to obtain reliable calculations, some convergence tests are performed to optimize the input parameters. Selfconsistency is achieved with the accuracy of $0.0001 \mathrm{eV}$ using the sufficient number of $k$-points for computations in the irreducible Brillouin zone. To study the effect of interatomic distances on the optical properties, the simplest case is chosen for the nanosystems; i.e. a free standing linear chain. Scheme of atomic positions in supercell, cell dimension labeled $d$, and also periodicity of linear and dimerized Ti nanochains is shown in Fig. 1.
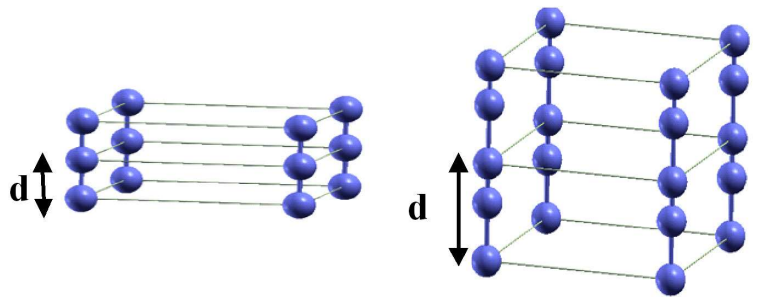

Fig. 1. Structures of linear (left) and dimerized (right) titanium nanochains ( $d$ is the length of cells).

Moreover, in order to obtain reliable calculations, we perform some convergence test to optimize the input parameters. The parameters, which have to be tested, are mainly $k$-points and $R_{\mathrm{mt}}-K_{\max }$. An optimized number of $k$-points values was only 700 for the dimerized nanochains and reached to 1500 points for the linear ones.

In combination of spherical harmonic inside no overlapping spheres surrounding the atomic sites, that are called muffin-tin (MT) spheres, and in a Fourier series in the interstitial region in the MT sphere, we expanded the basis function of $R_{\mathrm{mt}} K_{\max }$ for eigenvalues convergence.
$K_{\max }$ is the maximum modulus for the reciprocal lattice vector and $R_{\mathrm{mt}}$ is the average radius of the MT sphere. The convergence parameter $R_{\mathrm{mt}} K_{\max }$, which controls the size of the basis sets in this calculation is set to 8.5 for dimerized and 8 for linear nanochains of titanium.

More computational details such as the MT radii, atomic positions, and cohesive energy may be found in our previous work [37].

\section{Optical properties}

In both theory and experiment, the optical properties of metals and also insulators have been investigated by electromagnetic wave radiation on the surface material with the wave vector of $\boldsymbol{k}$ and frequency of $\omega$. Theoretically, by solving the Schrödinger equation in the presence of electromagnetic field and ignoring the nonlinear effect with the use of $a b$ initio approach, the function of optical properties is obtained. Also, the imaginary part of the frequency dependent dielectric function $\varepsilon_{2}(\omega)$ up to about $10 \mathrm{eV}$ using the expression given in our previous work [38]. The real part $\varepsilon_{1}(\omega)$ of the frequency-dependent dielectric function can be derived from the imaginary part using the Kramers-Kronig relation. From the real and imaginary parts of the frequencydependent dielectric function, the reflectivity $R(\omega)$, conductivity $\sigma(\omega)$, and electron energy loss $I(\omega)$ spectra can be calculated.

As the linear and dimerized $\mathrm{Ti}$ nanochains are more stable in nonmagnetic and ferromagnetic states, respectively, the optical properties of these atomic chains are studied in their stable state.

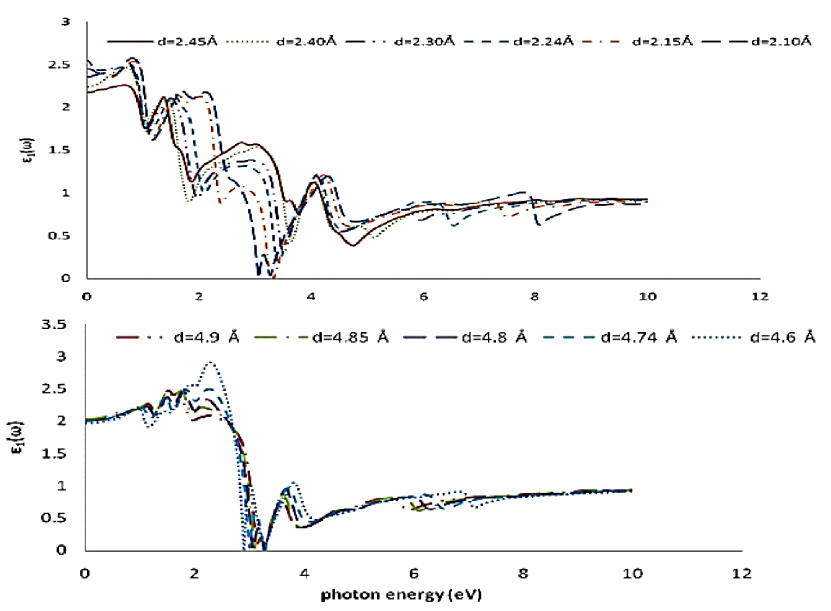

Fig. 2. Real parts of the dielectric function for linear (top) and dimerized (bottom) titanium nanochains at different lengths of cell $d(\AA)$.

In Fig. 2, the real part of dielectric functions of linear and dimerized titanium nanochains of different bond lengths is illustrated. Overall, $\varepsilon_{1}(\omega)$ is leveled off to the defined values of $\varepsilon_{1}(\infty)$ after some fluctuations. Also, $\varepsilon_{1}$ $(\omega)$ spectra are observed to shift to less energies, while the length of the structures is increased. Moreover, $\varepsilon_{1 \max }(\omega)$ 
decreases by increasing the atomic distances. Similarly, in the imaginary part of the dielectric function for these two structures (not shown here), all the peaks move toward less energies with increasing bond length.

Figure 3 shows the energy loss function for the linear and dimerized titanium nanochains. The plasma frequency (maximum value of energy loss function) for both structures decreases as the bond length increases. For the linear nanochains, this value is 2.4 at $d=2.1 \AA$ And decreases to 1.27 at $d=2.45 \AA$. Similarly, for dimerized nanochains, the plasma frequency is 2.2 and 1.2 at $d=4.6$ Aand $d=4.9 \AA$, respectively. Moreover, the peaks move toward higher energies with increasing bond length for linear structure, while they do not change significantly for the dimerized structure.

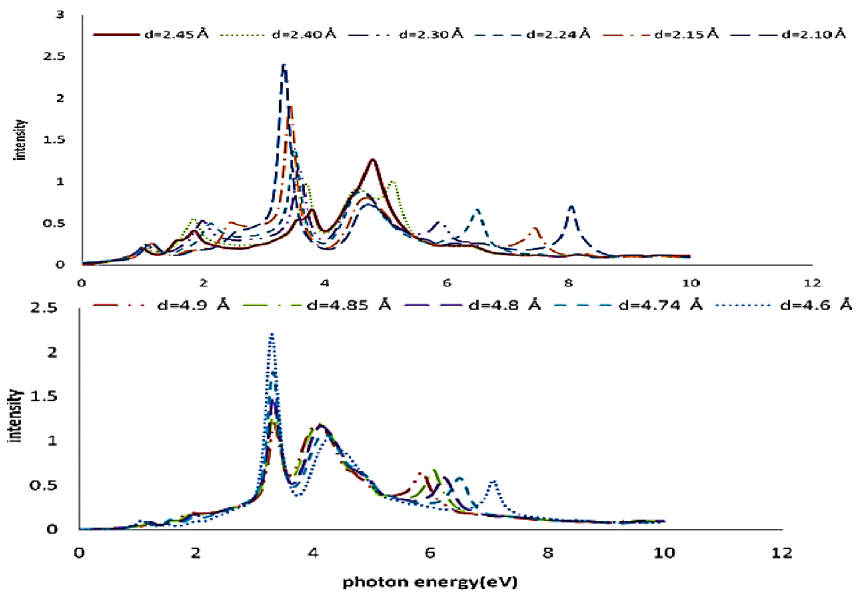

Fig. 3. Energy loss function for linear (top) and dimerized (bottom) titanium nanochains at different lengths of cell $d(\AA)$.

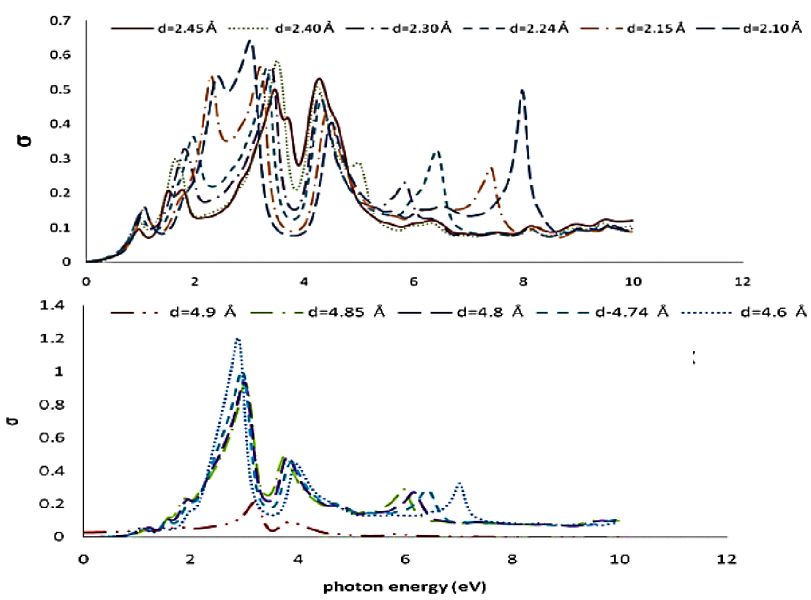

Fig. 4. Optical conductivity for linear (top) and dimerized (bottom) titanium nanochains at different lengths of cell $d(\AA)$.

The optical conductivity is determined according to the imaginary part of the dielectric function. In Fig. 4, this characteristic is calculated at different frequencies.
For both of the structures, the optical conductivity decreases with increasing the atomic distance. The maximum value of the optical conductivity for the linear and dimerized titanium nanochains is at $d=2.1$ Aand $d=4.6 \AA$, respectively, which are the minimum atomic distance in this investigation.

Figure 5 demonstrates the optical reflectivity of the two nanostructures. Under tensile strain of the atomic distances, this parameter decreases. The optical reflectivity spectra of dimerized nanochains slightly alter by changing the bond lengths.

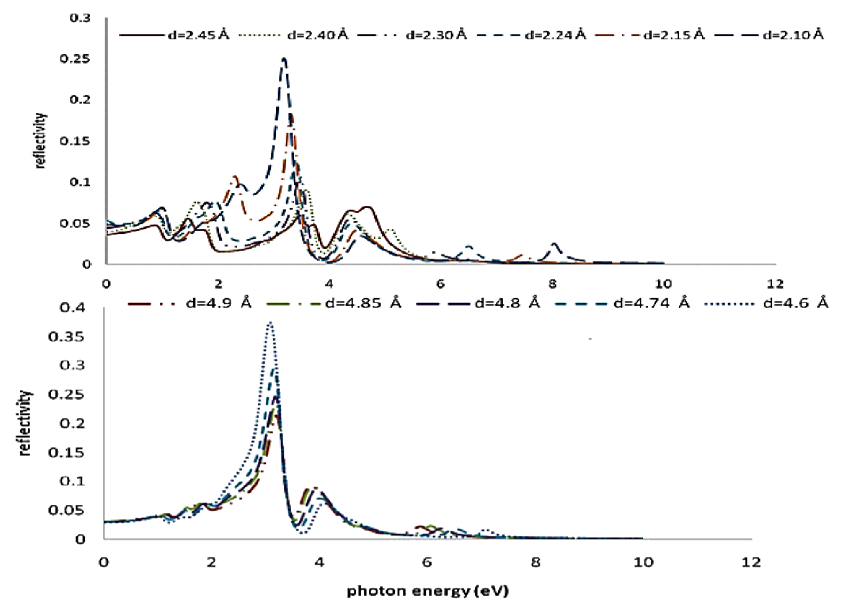

Fig. 5. Optical reflectivity for linear (top) and dimerized (bottom) titanium nanochains at different lengths of cell $d(\AA)$.

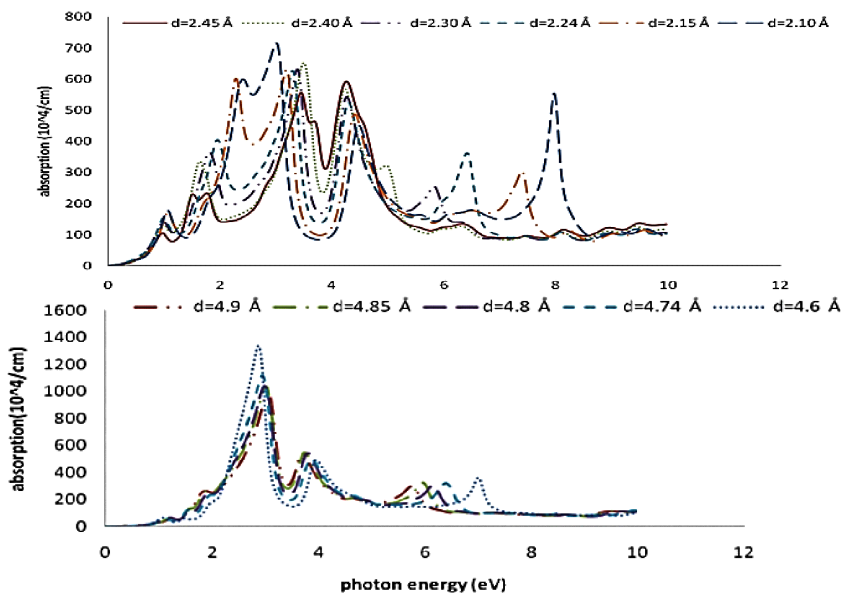

Fig. 6. Absorption coefficient for linear (top) and dimerized (bottom) titanium nanochains at different lengths of cell $d(\AA)$.

Finally, the absorption coefficient for the linear and dimerized titanium nanochains is calculated under different atomic distances in the photon energy range of 0 to $\approx 10 \mathrm{eV}$, as displayed in Fig. 6. A small absorption coefficient means that light is not absorbed by the material. Depth of penetration is defined by the distance at which the radiant decrease to $1 / \mathrm{e}$ of its incident value, in which $\alpha$ is the absorption coefficient. 
The fundamental absorption corresponds to a strong absorption region which is in the order of $10^{4}$ to $10^{5} \mathrm{~cm}^{-1}$. The fundamental absorption area is manifested by the rapid raise in absorption and is used for determining the energy gap of semiconductors. Figure 6 indicates that the absorption for both nanostructures decreases by increasing the bond lengths. These results also indicate that, under tensile strain, the absorption for linear nanochains moves toward higher energies.

\section{Conclusions}

In this paper, we have calculated the optical properties like dielectric function, loss function, optical conductivity, and reflectivity of linear and dimerized titanium nanochains using the LAPW method within GGA. Infinite and free standing periodic nanowires were considered with super cell approach. Peaks of the real part of the dielectric function were found to shift to the lower energies, while the length of the structures was increased. Moreover, the $\varepsilon_{1 \max }(\omega)$ decrease by increasing the atomic distances was investigated. It was shown that the plasma frequency for both structures decreased as the bond length increased and the optical conductivity decreased with increasing the atomic distances. It was observed that the maximum value of the optical conductivity for the linear and dimerized titanium nanochains occurs for the minimum atomic distance in this investigation. We also calculated the absorption coefficient for the linear and dimerized titanium nanochains and found out that for both nanostructures, this value decreases by increasing the bond lengths.

\section{References}

[1] A.I. Yanson, G.R. Bollinger, H.E. van den Bron, N. Agrait, J.M. van Ruitenbeek, Nature 395, 783 (1998).

[2] S. Ilani, L.A.K. Donev, M. Kindermann, P.L. McEuen, Nat. Phys. 2, 687 (2006).

[3] A. Colli, A. Fasoli, S. Pisana, Y. Fu, P. Beecher, W.I. Milne, A.C. Ferrari, Nano Lett. 8, 1358 (2008).

[4] M. Pratzer, H.J. Elmers, M. Bode, O. Pietzsch, A. Kubetzka, R. Wiesendanger, Phys. Rev. Lett. 87, 127201 (2001)

[5] R. Losio, K.N. Altmann, A. Kirakosian, J.L. Lin, D.Y. Petrovykh, F.J. Himpsel, Phys. Rev. Lett. 86 4632 (2001)

[6] J.C. Tung, G.Y. Guo, Phys. Rev. B 76, 094413 (2007).

[7] C. Ataca, S. Cahangirov, E. Durgun, Y.R. Jang, S. Ciraci, 0.1103/PhysRevB.77.214413Phys. Rev. B 77, 214413 (2008)

[8] A. Nakamura, K. Matsunaga, T. Yamamoto, Y. Ikuhara, Appl. Surf. Sci. 241, 38 (2005).

[9] G. Rubio, N. Agrait, S. Vieira, Phys. Rev. Lett. 76, 2302 (1996).

[10] Y. Yin, J. Jiang, Q. Cai, Appl. Surf. Sci. 199, 319 (2002).

[11] I. Mukhopadhyay, W. Freyland, Langmuir 19, 1951 (2003).
[12] P. Zhang, T.K. Sham, Phys. Rev. Lett. 90, 245502 (2003).

[13] G. Faraci, A.R. Pennisi, A. Balerna, H. Pattyn, G.E.J. Koops, G. Zhang, Phys. Rev. Lett. 86, 3566 (2001).

[14] J. Du, H. Wang, G. Jiang, J. Mol. Struct. Theochem. 817, 47 (2007).

[15] A. Pendelton, S. Kundu, H. Liang, J. Nanopart. Res. 11, 505 (2009).

[16] L. Zhang, P. Zhang, Y. Fang, J. Colloid Interface Sci. 311, 502 (2007).

[17] B.J. Inkson, G. Dehm, T. Wagner, J. Microsc. 214, $252(2004)$.

[18] Y.J. Chen, J.H. Hsu, H.N. Lin, Nanotechnology 16 , 1112 (2005)

[19] T. Miyazaki, K. Kopayashi, T. Horiuchi, H. Yamada K. Matsushige, Jpn. J. Appl. Phys. 40, 4365 (2001).

[20] Y. Zhang, H. Dai, Appl. Phys. Lett. 77, 3015 (2000).

[21] Y. Zhang, N.W. Franklin, R.J. Chen, H. Dai, Chem. Phys. Lett. 331, 35 (2000).

[22] B. Wang, S. Yin, G. Wang, J. Zhao, J. Phys. Condens. Matter 13, L403 (2001).

[23] A.Y. Li, R.Q. Li, Z.Z. Zhu, Y.H. Wen, Physica E 30 , 138 (2005).

[24] Z.L. Zhu, J.H. Gu, Y. Jia, Physica B Condens. Matter 387, 190 (2007).

[25] H. Hashemi, W. Hergert, V.S. Stepanyuk, J. Magn. Magn. Mater. 322, 1296 (2010).

[26] A. Bala, T. Nautiyal, J. Magn. Magn. Mater. 320 2201 (2008)

[27] A. Bala, T. Nautiyal, S. Auluk, J. Magn. Magn. Mater. 321, 1856 (2009).

[28] G. Grosso, G.P. Parravicini, Solid State Physics, Academic Press, Cambridge 2000.

[29] P. Hohenberg, W. Kohn, Phys. Rev. B 136, 864 (1964).

[30] P. Blaha, K. Schwarz, P. Sorantin, S.B. Rickey, Comp. Phys. Commun. 59, 399 (1990).

[31] P. Blaha, K. Schwarz, G.K.H. Madsen, D. Kvasnicka, J. Luitz, WIEN2K, An Augmented Plane Wave Plus Local Orbitals Pogram for Calculating Crystal Properties, Vienna University of Technology, Vienna, Austria 2001

[32] J.P. Perdew, Y. Wang, Phys. Rev. B 45, 13244 (1992).

[33] J.P. Perdew, J.A. Chevary, S.H. Vosko, K.A. Jackson, M.R. Pederson, D.J. Singh, C. Fiolhais, Phys. Rev. B 46, 6671 (1992).

[34] J.P. Perdew, K. Burke, M. Ernzerhof, Phys. Rev. Lett. 77, 3865 (1996).

[35] J.P. Perdew, K. Burke, M. Ernzerhof, Phys. Rev Lett. 78, 1396 (1997).

[36] J.P. Perdew, S. Kurth, A. Zupan, P. Blaha, Phys. Rev. Lett. 82, 2544 (1999)

[37] M. Jafari, H. Hajiyani, Z. Sohrabikia, H. Galavani, Comput. Mater. Sci. 77, 224 (2013).

[38] M. Jafari, Z. Sohrabikia, H. Galavani, Phys. Scr 86, 055602 (2012). 